\title{
Digestibilidad de dietas con niveles crecientes de harina de coco desgrasada en conejos en crecimiento
}

\section{Digestibility of diets with growing levels of defatted coconut flour in growing rabbits}

\author{
Yanixi Acosta-Acosta ${ }^{1}$ \\ Ángel Luis La-O-Michel ${ }^{2}$ \\ Fecha de recepción: 22 de mayo de 2017 \\ Fecha de aprobación: 25 de agosto de 2017 \\ Manuel Isidoro Valdivié-Navarro ${ }^{3}$ \\ Jaqueline Cantalapiedra-Bello ${ }^{4}$
}

DOI: https://doi.org/10.19053/01228420.v15.n1.2018.7755

\section{Resumen}

Para evaluar la influencia de la harina de coco desgrasada sobre los coeficientes de digestibilidad aparente (CDA) de los nutrientes para conejos en crecimiento, fueron elaboradas cinco dietas con niveles crecientes de esta $(0,10,20,30$ y $40 \%)$, las cuales fueron suministradas a 50 animales de la raza Chinchilla, con un diseño completamente aleatorizado. Fueron determinados los CDA de la Materia seca (MS), Proteína bruta (PB), Fibra bruta (FB), Materia orgánica (MO) y las Cenizas (Czs). El período experimental fue de 12 días. La inclusión de la harina de coco desgrasada en la dieta no afectó el CDA de la MS, con valores promedios próximos al $60 \%$. Se encontraron altos CDA para la PB (74.92 y $77.86 \%$ ), los cuales decrecieron para los niveles de inclusión del $30 \%$ y el $40 \%$. Los CDA de la FB presentaron tendencia similar a los de la PB, con valores medios entre 44.66 y $49.80 \%$. Los conejos mostraron un buen aprovechamiento del contenido de cenizas, con CDA de $66.80 \%$ a $72.99 \%$, superiores para los niveles de inclusión del $0 \%$ y $10 \%$. Los resultados indican que los conejos realizaron una elevada absorción de los compuestos inorgánicos.

Palabras clave: animales monogástricos; cunicultura; subproducto del coco; utilización digestiva de los nutrientes.

1 Ph. D. Universidad de Guantánamo (Guantánamo, Cuba). yanixi@cug.co.cu.

2 Ph. D. Universidad de Guantánamo (Guantánamo, Cuba).nolo@cug.co.cu.

3 Ph. D. Instituto de Ciencia Animal (Mayabeque. Cuba).mvaldivie@ica.co.cu.

4 M. Sc. Universidad de Guantánamo (Guantánamo, Cuba).yaque@cug.co.cu. 


\begin{abstract}
In order to evaluate the influence of defatted coconut flour on the apparent digestibility coefficients (ADC) of nutrients for growing rabbits, five diets were elaborated with increasing levels of defatted coconut flour $(0 \%, 10 \%, 20 \%, 30 \%$ and $40 \%)$. The diets were given to 50 Chinchilla rabbits, distributed in a totally randomized design, with an experimental period of 12 days. The ADC of the dry matter (MS), gross protein $(\mathrm{PB})$, gross fiber (BFC), organic matter $(\mathrm{MO})$ and ash (Czs) were determined. The inclusion of defatted coconut flour into the rabbit's diet had not effect on the ADC of MS, with average values close to $60 \%$. High values of ADC of PB were observed (74.92\% and $77.86 \%$ ), which decreased for levels of defatted coconut flour of $30 \%$ and $40 \%$. The ADC of BFC behaved similar to the PB, with mean values between $44.66 \%$ and $49.80 \%$. The rabbits showed a good use of the ash content with ADC from 66.80 $\%$ to $72.99 \%$, which were more elevated for diets with $0 \%$ and $10 \%$ of defatted coconut flour. The results indicate that the rabbits carried out a high absorption of the inorganic compounds.
\end{abstract}

Keywords: coconut byproduct; cuniculture; digestive use of nutrients; monogastrics animals.

\title{
Para citar este artículo:
}

Acosta-Acosta Y., La-O-Michel AL., Valdivié-Navarro MI., Cantalapiedra-Bello J. Digestibilidad de dietas con niveles crecientes de harina de coco desgrasada en conejos en crecimiento. Ciencia y Agricultura. 2018; 15(1): 45-51. 


\section{Introducción}

Actualmente, son materia de estudio gran cantidad de residuos agrícolas, con el fin de emplearlos como fuentes alternativas en la alimentación animal que generen rentabilidad. En Cuba, específicamente en la provincia de Guantánamo, los residuos más abundantes son la pulpa de café, la cáscara de cacao y la harina de coco, los cuales generan problemas ambientales que pueden ser reducidos con el establecimiento de explotaciones cunícolas, bien sea a nivel doméstico o industrial, que a su vez representen una fuente de ingresos para los productores de la región.

La harina de coco desgrasada, residuo obtenido de la extracción del aceite de coco por medio de solvente o prensado, es un alimento rico en fibra que, precisamente por esto, puede ser usado en raciones de conejos, ya que las dietas para estos animales demandan altos tenores de fibra. La proteína de la harina de coco es de calidad superior a la del maíz, y por la deficiencia en lisina, su valor proteico es inferior a la harina de soya. A pesar de que la proteína encontrada en este alimento sea inferior a las materias primas usadas normalmente como suplemento proteico, su uso puede ser económicamente viable en áreas donde la disponibilidad de otras fuentes de proteína de calidad sea escasa (1).

En vista de desarrollar programas alimenticios para conejos de manera más eficiente, utilizando estos residuos, es necesario tener conocimiento de sus cualidades específicas para esta especie, incluyendo digestibilidad, palatabilidad, contenido nutricional y los efectos sobre el funcionamiento intestinal y las enfermedades entéricas (2). El objetivo del presente trabajo fue determinar los coeficientes de digestibilidad aparente de los nutrientes de dietas contentivas de niveles crecientes de harina de coco desgrasada industrialmente.

\section{Materiales y métodos}

El experimento fue realizado en el polígono docente investigativo del Centro de Estudio de Tecnología Agropecuaria de la Facultad Agroforestal, perteneciente a la Universidad de Guantánamo. Se registraron diariamente los datos de temperatura y humedad relativa del interior de la instalación. Las temperaturas medias, máximas y mínimas y la humedad relativa del aire durante el experimento fueron $30.4 \pm 0.9^{\circ} \mathrm{C} ; 28.1 \pm 1.2^{\circ} \mathrm{C}$ e $70 \pm 2 \%$, respectivamente.

Fueron utilizados 50 animales de 75 días de edad, de la raza Chinchilla, con peso promedio de $2337 \mathrm{~g}$, ubicados individualmente en jaulas de metabolismo bajo un diseño completamente aleatorizado; las jaulas, de $0.16 \mathrm{~cm}^{2}$ y con bebederos y comederos, fueron construidas con alambre galvanizado; en el fondo de ellas fueron colocados colectores de excretas construidos con mallas plásticas. Los tratamientos fueron constituidos por una ración referencial y cuatro raciones por investigar, con $10 \%, 20 \%, 30 \%$ y $40 \%$ de inclusión de harina de coco desgrasada. Las variables evaluadas fueron el consumo de materia seca (MS) y los coeficientes de digestibilidad aparente (CDA) de la materia seca $(\mathrm{MS})$, proteína bruta (PB), materia orgánica $(\mathrm{MO})$, fibra bruta $(\mathrm{FB})$ y la ceniza (Czs).

El experimento se realizó durante 12 días; los siete primeros fueron de adaptación, y los cinco restantes, de recolección de heces. Los animales fueron pesados al inicio y al final del experimento. Durante la fase de adaptación, los alimentos fueron ofertados a voluntad, y durante la fase experimental se ofertaron 140 g.animal.día ${ }^{-1}$; los animales tuvieron acceso al agua a voluntad.

Las dietas fueron elaboradas con base en las exigencias nutricionales de los animales reportados por De Blas y Mateos (3); las mezclas de las materias primas se realizaron de forma manual y peletizadas en una máquina de fabricación criolla, garantizando la homogeneidad del alimento, conforme al volumen de alimento procesado. El suministro de alimento fue realizado en la mañana y en la tarde. Los valores de la composición porcentual de las dietas se presentan en la Tabla I. 
Tabla I. Composición porcentual de los ingredientes en las dietas evaluadas.

\begin{tabular}{|l|c|c|c|c|c|}
\hline \multirow{2}{*}{ Ingredientes } & \multicolumn{5}{|c|}{ Niveles de inclusión de harina de coco desgrasada } \\
\cline { 2 - 5 } & $\mathbf{0} \%$ & $\mathbf{1 0} \%$ & $\mathbf{2 0} \%$ & $\mathbf{3 0} \%$ & $\mathbf{4 0} \%$ \\
\hline Harina de maíz & 24.72 & 23.99 & 23.05 & 16.65 & 7.8 \\
\hline Harina de alfalfa & 55.33 & 50.04 & 45.84 & 46.33 & 47.96 \\
\hline Harina de soya & 12.87 & 10.11 & 6.85 & 2.85 & 0 \\
\hline Harina de coco industrial & 0.00 & 10.00 & 20 & 30 & 40 \\
\hline Fosfato di-cálcico & 1.06 & 0.90 & 0.73 & 0.58 & 0.45 \\
\hline Carbonato calcio & 0.00 & 0.00 & 0.00 & 0.00 & 0.17 \\
\hline Sal común & 0.30 & 0.30 & 0.30 & 0.30 & 0.30 \\
\hline Metionina & 0.12 & 0.12 & 0.12 & 0.12 & 0.12 \\
\hline Lisina & 0.00 & 0.04 & 0.11 & 0.17 & 0.20 \\
\hline Premezcla & 2.00 & 2.00 & 2.00 & 2.00 & 2.00 \\
\hline Zeolita & 1.50 & 1.00 & 1.00 & 1.00 & 1.00 \\
\hline Aceite de coco & 2.10 & 1.50 & 0 & 0 & 0 \\
\hline
\end{tabular}

La recolección de heces se realizó diariamente, a las 8:00 a.m.; estas se almacenaron en bolsas plásticas, $\mathrm{a}-10^{\circ} \mathrm{C}$, y al final fueron descongeladas a temperatura ambiente, se homogeneizaron y pesaron y se tomaron muestras para las determinaciones analíticas.

\section{A. Determinaciones analíticas}

$$
C D A=\frac{\text { Total de nutriente concumido }- \text { total de nutriente excretado }}{\text { Total de nutriente consumido }} \times 100
$$

\section{B. Análisis estadístico}

Los valores del coeficiente de digestibilidad aparente de los diferentes nutrientes y los indicadores fueron sometidos a un análisis de varianza simple; las diferencias entre las medias se determinaron por la prueba de Duncan (7). En todos los casos se utilizó el paquete estadístico Statgraphics plus 5.1.

\section{Resultados y discusión}

En la Tabla II se aprecia la similitud en cuanto al contenido nutritivo de las dietas elaboradas con la inclusión de la harina de coco desgrasada como materia prima; la materia seca (MS) mantuvo valores superiores al $90 \%$, lo cual sugiere una adecuada concentración de nutrientes.
Los contenidos de materia seca, cenizas, materia orgánica y proteína bruta se determinaron a partir de la metodología descrita por la AOAC (4), y el contenido de fibra bruta, según Van Soest et al. (5). De los resultados obtenidos se calculó el CDA, la MS, la FB, la PB, la MO y la Czs, a partir de la ecuación sugerida por Matterson et al. (6), donde:
El contenido de PB fue de alrededor del $16 \%$, y el de FB, del $14 \%$; ambos nutrientes en la dieta están en correspondencia con las recomendaciones para esta especie informadas por Ferreira et al. (8), quienes reportan un rango que oscila entre el $16 \%$ y el $18 \%$ de $\mathrm{PB}$, y por De Blas y Mateos (9), quienes recomiendan $\mathrm{PB}$ entre $14.5 \%$ y $16.2 \%$ y FB de $13.5 \%-15.0 \%$, así como con las informadas más recientemente por De Blas y Mateos (3), de $14.2 \%-16.0 \%$ para la $\mathrm{PB}, \mathrm{y}$ rango superiores de $15.0-16.0 \%$ para la FB.

Las dietas elaboradas exhibieron un alto contenido de Czs, con rango de 11.03-12.45\%, lo cual sugiere que pudieran tener un adecuado balance de minerales, capaz de suplir las exigencias de los conejos para estos componentes. 
Tabla II. Composición química de dietas con niveles crecientes de harina de coco desgrasada en conejos adultos.

\begin{tabular}{|l|c|c|c|c|c|}
\hline \multirow{2}{*}{ Nutrientes } & \multicolumn{5}{|c|}{ Niveles de inclusión de la harina de coco desgrasada } \\
\cline { 2 - 6 } & $\mathbf{0} \%$ & $\mathbf{1 0} \%$ & $\mathbf{2 0} \%$ & $\mathbf{3 0} \%$ & $\mathbf{4 0} \%$ \\
\hline Materia seca & 92.08 & 91.13 & 90.57 & 91.3 & 91.61 \\
\hline Proteína bruta & 16.04 & 16.02 & 16.00 & 16.03 & 16.00 \\
\hline Fibra bruta & 14.08 & 14.43 & 14.09 & 14.69 & 13.97 \\
\hline Cenizas & 12.45 & 12.99 & 11.30 & 11.03 & 12.61 \\
\hline Materia orgánica & 87.55 & 87.01 & 88.70 & 88.97 & 87.39 \\
\hline
\end{tabular}

El consumo de MS está en correspondencia con la edad y peso de los animales; no obstante, se observó que los conejos alimentados con las dietas con $10 \%$ y $20 \%$ de harina de coco desgrasada mostraron consumo significativamente superior al del grupo de animales alimentados con la dieta con $0 \%$, mientras aquellos que consumieron dietas con $30 \%$ y $40 \%$ no presentaron diferencias respecto del grupo que consumió la dieta referencial.
Vasconcelos (1), utilizando niveles de $6.25 \%, 12.5$ $\%, 18.25 \%$ y $25 \%$ de harina de coco en la dieta de conejos, encontró tendencia similar a la de este trabajo; observó que los animales alimentados con ración que contenía 18,75 \% de harina de coco presentaban consumo significativamente mayor al de aquellos alimentados con dietas sin harina de coco, mientras que aquellos alimentados con ración con el $25 \%$ de harina de coco consumieron menos que el grupo control.

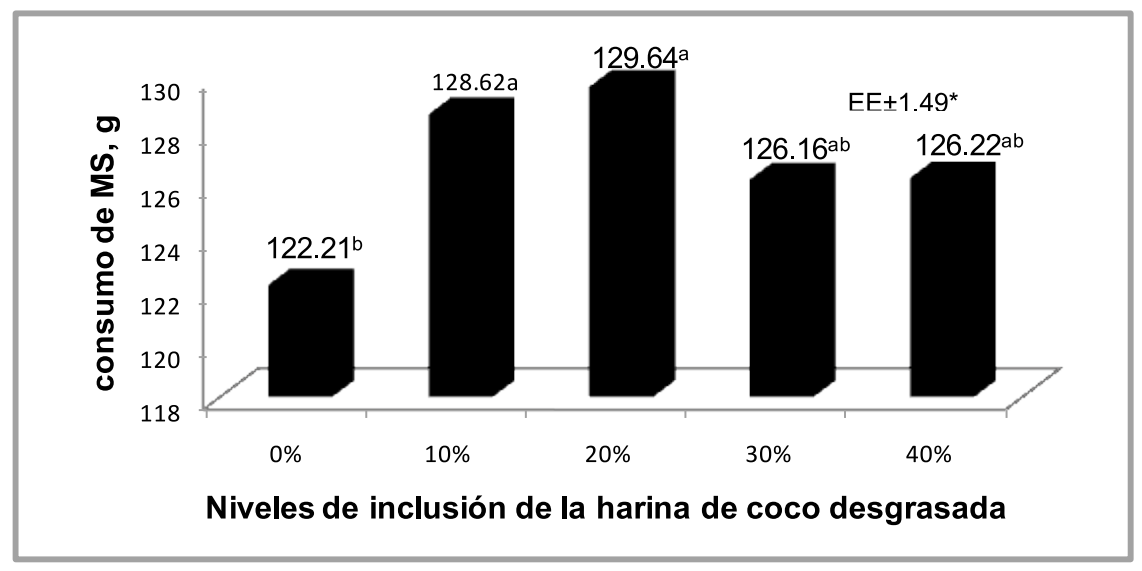

Fig. 1. Consumo de materia seca de los conejos de dieta con niveles creciente de harina de coco desgrasada.

En la Tabla III se presentan los CDA de MS, PB, $\mathrm{FB}, \mathrm{Czs}$ y $\mathrm{MO}$; se observa que la inclusión de la harina de coco desgrasada en la dieta no afectó el coeficiente de digestibilidad de la MS, con valores promedios próximos al $60 \%$, similares a los reportados por Fierro (10) en una evaluación nutritiva de piensos comerciales para conejos, donde encontró CDA de la MS entre 57.69 y 60.81 , y superiores al $45 \%$ encontrado por Vasconcelos (1) utilizando nivel de $6.25 \%, 12.5 \%, 18.25 \%$ y $25 \%$. En estos valores de digestibilidad de la MS es probable que haya contribuido el contenido de $\mathrm{FB}$. Al respecto, Faria et al. (11) señalan que la fibra vegetal regula la velocidad de tránsito digestivo, dificultando o mejorando la acción enzimática en el intestino delgado. Por su parte, Quadros et al. (12) relatan que en la digestibilidad es necesario hacer distinción entre las fibras, en función de su composición química; un ejemplo es la cáscara de soya y el afrecho de trigo, los cuales son fibrosos, pero la cáscara de soya tiene gran cantidad de pectina, que es altamente digestible. 
Los CDA de la PB se encontraron altos para los niveles de inclusión de la harina de coco desgrasada en las dietas; sus valores oscilaron entre $74.92 \%$ y $77.86 \%$, y hubo diferencias con respecto a la dieta referencial para los niveles de inclusión del $30 \%$ y el $40 \%$; resultados similares encontró Vasconcelos (1), con CDA de la PB del $80.65 \%$, y fueron superiores a los reportados por Josianny Limeira (13), utilizando cáscara de soya molida ensilada con inoculante y sin inoculante con \% de digestibilidad de $55.0 \%$, $57.5 \%$ y $57.13 \%$.

Investigando diferentes fuentes de fibra y almidón para conejos en crecimiento, con dietas que contenían cáscara de soya, Arruda et al. (14) reportaron valores de CDA para la PB de $69.10 \%$, similares a los obtenidos en el presente trabajo. Quadros et al. (12) observaron que el ensilaje de cáscara de soya molida mejoró la digestibilidad de la PB (68.53 vs. 59.44).

Los CDA de la FB presentaron tendencia similar a los de la $\mathrm{PB}$, con valores medios entre 44.66 y 49.80, superiores al $33.77 \%$ encontrado por Scapinello et al. (15), quienes utilizaron heno de rama de yuca; esto confirma lo señalado por Vasconcelos (1), quien encontró coeficientes de digestibilidad de $63.73 \%$ para la FB de la harina de coco y los atribuyó a la elevada proporción de fibra digestible que esta presenta.

Los conejos mostraron un buen aprovechamiento del contenido de cenizas, con CDA de $66.80 \%$ a $72.99 \%$; los mayores valores para los niveles de inclusión del $0 \%$ y $10 \%$. Los resultados indican que los conejos realizaron una elevada absorción de los compuestos inorgánicos presentes en las dietas; al respecto, De Blas y Mateos (9) señalan que la absorción de estos compuestos por los conejos es muy eficiente, independientemente de la necesidad del animal.

Los CDA para la MO fueron mayores para el caso de la dieta referencial, y entre las dietas que incluyeron la harina de coco desgrasada no hubo diferencias, y en todos los casos fueron inferiores a los reportados por Martin et al. (16), quienes informaron valores de $80.68 \%$, utilizando dietas con diferentes niveles de almidón, resultados que atribuyeron a la inclusión de elevadas cantidades de fuentes energéticas. Los valores obtenidos en el presente trabajo fueron superiores a los reportados por Villamide y De Blas (17) al evaluar dietas con, aproximadamente, $23 \%$ de almidón, con digestibilidades medias de $60 \%$.

Tabla III. CDA de los nutrientes de dietas con niveles crecientes de harina de coco desgrasada en conejos adultos.

\begin{tabular}{|l|c|c|c|c|c|c|}
\hline \multirow{2}{*}{ Nutrientes } & \multicolumn{5}{|c|}{ Niveles de inclusión de la harina de coco desgrasada } & \multirow{2}{*}{ EE, sig } \\
\cline { 2 - 6 } & $\mathbf{0 \%}$ & $\mathbf{1 0} \%$ & $\mathbf{2 0} \%$ & $\mathbf{3 0} \%$ & $\mathbf{4 0} \%$ & \\
\hline Materia seca & 58.92 & 58.42 & 57.56 & 58.50 & 58.59 & $0.99^{\text {ns }}$ \\
\hline Proteína bruta & $77.86^{\mathrm{a}}$ & $76.91^{\mathrm{ab}}$ & $76.56^{\mathrm{abc}}$ & $75.99^{\mathrm{bc}}$ & $74.92^{\mathrm{c}}$ & $1.01^{*}$ \\
\hline Fibra bruta & $49.80^{\mathrm{a}}$ & $48.33^{\mathrm{ab}}$ & $47.59^{\mathrm{abc}}$ & $46.13^{\mathrm{bc}}$ & $44.66^{\mathrm{c}}$ & $1.14^{*}$ \\
\hline Cenizas & $72.99^{\mathrm{a}}$ & $71.48^{\mathrm{a}}$ & $68.116^{\mathrm{b}}$ & $67.52^{\mathrm{b}}$ & $66.80^{\mathrm{b}}$ & $0.69^{*}$ \\
\hline Materia orgánica & $74.12^{\mathrm{a}}$ & $72.01^{\mathrm{b}}$ & $71.92^{\mathrm{b}}$ & $71.80^{\mathrm{b}}$ & $71.55^{\mathrm{b}}$ & $0.56^{*}$ \\
\hline
\end{tabular}

${ }^{a b c}$ superíndices diferentes en la misma fila indican diferencias significativas a $p \leq 0.05$

\section{Conclusiones}

Las dietas elaboradas con la inclusión de $10 \%$, $20 \%, 30 \%$ y $40 \%$ de harina de coco desgrasada mantuvieron un balance de nutrientes adecuado para la especie, con valores de $16 \%$ de PB y de 13.97 a $14.69 \%$ de FB.
El consumo de alimento mostró valores entre 122.21 g.animal. $d^{-1}$ y 129.64 g.animal.d ${ }^{-1}$; se observó que la inclusión de $10 \%$ y $20 \%$ de harina de coco en las dietas promovió un incremento del consumo de alimentos.

La inclusión de la harina de coco desgrasada en las dietas, en los niveles de $10 \%, 20 \%, 30 \%$ y 
$40 \%$, no afectó los CDA de la MS. Para la PB y la FB hubo una tendencia a disminuir respecto a la dieta referencial en las inclusiones del $30 \%$ y el $40 \%$, mientras para la Czs y la $\mathrm{MO}$ el descenso se mostró a partir del $20 \%$ y el $10 \%$ de inclusión, respectivamente.

\section{Referencias}

(1) Vasconcelos CA. Utilização do Farelo de Côco em dietas para coelhos destinados ao abate. Dissertação de Mestrado. Fortaleza-Ceará. Universidade Federal do Ceará Centro de Ciências Agrárias. Programa de Pós-graduação em Zootecnia. 2007.

(2) Valverde DM. Usos de la morera (Morus alba) en la alimentación del conejo: El rol de la fibra y la proteína en el tracto digestivo. Agronomía Mesoamericana. 2010; 21(20): 357-366.

(3) De Blas C., Mateos GG. Feed formulation. Ed: Blas C., Wliseman J. In: Nutrition of the rabbit. Cambridge: CAB International. 2010: 222-232. DOI: http://doi.org/10.1079/9781845936693.0222.

(4) Association Official Analytical Chemistry-AOAC. Official methods of analysis. 11 ed. Washington DC. 1995.

(5) Van Soest PJ., Robertson JB., Lewis BA. Methods for dietary fiber, neutral detergent fiber and nonstarch polysaccharides in relation to animal nutrition. J. Dairy Sci. 1991; 74: 3583-3597. DOI: http://doi.org/10.3168/jds.S0022-0302(91)78551-2.

(6) Matterson LD., Potter LM., Stutz NW., Singsen EP. The metabolizable energy of feed ingredients for chickens. Storrs: The University of Connecticut; Agricultural Experiment Station. 1965.

(7) Duncan B. Multiple Range and Multiple F. Test. Biometrics, 1955; 11: 1-7. DOI: http://doi.org/10.2307/3001478.

(8) Ferreira VPA., Ferreira WM., Saliba SEO., Scapinello C., Teixeira AO., Kamwa EB. Digestibilidade, cecotrofia, desempenho e rendimento de carcaça de coelhos em crescimento alimentados com rações contendo óleo vegetal ou gordura animal. Revista Brasileira de Zootecnia. 2006; 35(4): 1696-1704. DOI: http:// doi.org/10.1590/S1516-35982006000600017.

(9) De Blas JC., Mateos GG. The nutrition of the rabbit. Cambridge: CAB International. 1998: 241-253.

(10) Fierro JF. Valoración nutritiva de piensos comerciales para conejos. Máster de Zootecnia y Gestión Sostenible: Ganadería Ecológica e Integrada de la Universidad de Córdoba. 2014: 1-11.

(11) Faria HG., Ferreira WM., Scapinello C., Oliveira CEA. Efeito da utilização de dietas simplificadas, à base de forragem, sobre a digestibilidade e desempenho de coelhos Nova Zelândia. Revista Brasileira de Zootecnia. 2008; 37(10): 1797-1801. DOI: http:// doi.org/10.1590/S1516-35982008001000012.

(12) Quadros ARB., Moreira I., Paiano D. Avaliação nutricional da casca de soja integral ou moída, ensilada ou não, para suínos na fase de crescimento. Acta Scientiarum. Animal Sciences. Maringa. 2007; 29(1): 31-38.

(13) Josianny L. Casca de soja na alimentação de coelhos em crescimento em substituição aos fenos de alfafa e de Cost cross. Dissertação apresenta, como parte das exigências para a obtenção do título de Mestre em zootecnia. Universidade estadual de Maringá. Centro de ciências agrárias. 2009.

(14) Arruda AMV., López DC., FerriraWM. Digestibilidade aparente dos nutrientes de rações contendo diferentes fontes de fibra e níveis de amido com coelhos em crescimento. Revista Brasileira e Zootecnia. Viçaosa. 2002; 31(3).

(15) Scapinello C., Falco JE., Furlan AC., Faria HG. Valor nutritivo do feno da rama da mandioca (Manihot esculenta, Crantz) para coelhos em crescimento. Revista Brasileira de Zootecnia. 1999; 28(5): 1063-1067. DOI: http://doi.org/10.1590/S151635981999000500023.

(16) Martins A., Dessimoni R., Gonçalves R. Desempenho Produtivo e Atividade Microbiana Cecal de Coelhos Alimentados com Dietas Contendo Diferentes Níveis de Amido. Rev. Bras. Zootec. 2000; 29(3): 762-768. DOI: http://doi.org/10.1590/S151635982000000300018.

(17) Villamide MJ., De Blas C. Nutritive value of cereal grains for rabbits. Journal Appl. Rabbit Research. 1991; 14(2): 144-147. 
